University of Nebraska - Lincoln

DigitalCommons@University of Nebraska - Lincoln

6-1-2006

\title{
Molecular simulations of solid-liquid interfacial tension of silicon
}

\author{
Yuk Wai Tang \\ University of Nebraska-Lincoln, ytang2@Unl.edu \\ Jun Wang \\ University of Nebraska-Lincoln, jwang3@unl.edu \\ Xiao Cheng Zeng \\ University of Nebraska-Lincoln, xzeng1@unl.edu
}

Follow this and additional works at: https://digitalcommons.unl.edu/chemzeng

Part of the Chemistry Commons

Tang, Yuk Wai; Wang, Jun; and Zeng, Xiao Cheng, "Molecular simulations of solid-liquid interfacial tension of silicon" (2006). Xiao Cheng Zeng Publications. 7.

https://digitalcommons.unl.edu/chemzeng/7

This Article is brought to you for free and open access by the Published Research - Department of Chemistry at DigitalCommons@University of Nebraska - Lincoln. It has been accepted for inclusion in Xiao Cheng Zeng Publications by an authorized administrator of DigitalCommons@University of Nebraska - Lincoln. 


\title{
Molecular simulations of solid-liquid interfacial tension of silicon
}

\author{
Yuk Wai Tang, Jun Wang, and X. C. Zeng ${ }^{\text {a) }}$ \\ Department of Chemistry, University of Nebraska-Lincoln, Lincoln, Nebraska 68588
}

(Received 23 March 2006; accepted 25 April 2006; published online 20 June 2006)

[DOI: $10.1063 / 1.2206592]$

Silicon is an important semiconductor for manufacturing of microelectronic chips used in computers and other electronic devices. During the manufacturing process, liquid silicon is being solidified to make high-grade solid semiconductors. Thus, improved data of the solid-liquid interfacial properties of silicon will be useful towards optimizing the experiment condition for making better quality electronics. Yet it is difficult to perform experiments of liquid silicon due to its high melting point. Thus far, most experimental measurements of the solid-liquid interfacial tension are based on the measurement of rate of homogeneous crystal nucleation at a given undercooling. The first measurement of solidliquid interfacial tension of silicon was performed by Stiffler et $a l .{ }^{1}$ Following pulsed-laser melting thin films of silicon, a deep undercooling of $505 \mathrm{~K}$ was achieved. The measured rate of nucleation is about $10^{29}$ events $/ \mathrm{s} \mathrm{m}^{3}$, which yields a solid-liquid interfacial tension $\gamma_{\mathrm{sl}}=0.34 \pm 0.02 \mathrm{~J} / \mathrm{m}^{2}$. The estimation of surface tension is based on the assumptions that the critical nucleus is spherical and the nucleation is homogeneous.

Besides thin films of silicon, deep undercooling has also been achieved in bulk liquid silicon using containerless processing techniques, such as electrostatic levitation, ${ }^{2}$ flux processing, ${ }^{3}$ and electromagnetic levitation. ${ }^{4-6}$ Shao and Spaepen $^{3}$ processed pure $\mathrm{Si}$ drops about $1 \mathrm{~mm}$ in diameter surrounded by a $\mathrm{SiO}_{2}-\mathrm{BaO}-\mathrm{CaO}$ flux and achieved a maximum undercooling of $350 \mathrm{~K}$. This undercooling is much deeper than that $(270 \mathrm{~K})$ of the first undercooling experiment with quartz tubes. ${ }^{7}$ Again, based on the classical theory of nucleation, the estimated $\gamma_{\mathrm{sl}} \sim 0.38 \mathrm{~J} / \mathrm{m}^{2}$, slightly higher than $0.34 \mathrm{~J} / \mathrm{m}^{2}$ from the laser-melting measurement. Using electromagnetic levitation method, ${ }^{4} \mathrm{Li}$ and Herlach achieved the deepest bulk undercooling of $420 \mathrm{~K}$ in liquid $\mathrm{Si}$ (with the drop size of about $7 \mathrm{~mm}$ in diameter). Similar experiments were performed by Liu et al. for drop size of $10 \mathrm{~mm}$ and an undercooling up to $330 \mathrm{~K}$ was produced. ${ }^{6}$ The estimated $\gamma_{\mathrm{sl}} \sim 0.40 \mathrm{~J} / \mathrm{m}^{2}$

Few theoretical studies of silicon solid-liquid interfacial properties have been reported to date. Earlier, a theoretical result $\gamma_{\mathrm{sl}}=0.167 \mathrm{~J} / \mathrm{m}^{2}$ was reported using Ginzburg-Landau theory. ${ }^{8}$ In contrast, several computational studies of liquidvapor surface tension of silicon have been reported. Wang and Stroud reported liquid-vapor surface tension of silicon by evaluation of free energy using Monte Carlo simulations.' Another report recently was based on molecular simulations of bulk systems with reversible scaling method. ${ }^{10}$ Using a superheating/undercooling method in molecular simulation, ${ }^{11}$ Luo et al. simulated solid-liquid transitions of a Lennard-
Jones system ${ }^{12}$ as well as water/ice system $^{13}$ and reported their solid-liquid interfacial tensions. In this short note, we apply the simulation method of Luo et al. ${ }^{11-13}$ to calculate solid-liquid interfacial tension of silicon.

The principle of superheating and undercooling method is based on classical nucleation theory ${ }^{11}$ where the highest temperature achieved by superheating a solid $\left(T^{+}\right)$or the lowest temperature by undercooling $\left(T^{-}\right)$depend on a dimensionless nucleation barrier parameter $(\beta)$ and the heating rate $(Q) . \beta$ is calculated by the equation

$$
\beta=\left(A_{0}-b \log _{10} Q\right) \theta_{c}\left(\theta_{c}-1\right)^{2},
$$

where $A_{0}=59.4, b=2.33$, and $Q$ is normalized by $Q_{0}$ $=1 \mathrm{~K} / \mathrm{s}$. The dimensionless temperature $\theta_{c}=T^{+} / T_{m}$ or $T^{-} / T_{m}$, where $T_{m}$ is the melting temperature. In the case of silicon, $T^{-}$is much more difficult to determine because of the transition of silicon from liquid to amorphous under the fast cooling rate in molecular dynamics (MD) simulations. ${ }^{14-17}$ Hence, we calculate $\beta$ by using only the superheating temperature and the melting point of respective silicon model. Once $\beta$ is obtained, solid-liquid interfacial tension $\left(\gamma_{\mathrm{sl}}\right)$ can be calculated by

$$
\gamma_{\mathrm{sl}}=\left(\frac{3}{16 \pi} \beta k_{B} T_{m} \Delta H_{m}^{2}\right)^{1 / 3},
$$

where $\Delta H_{m}$ is the enthalpy change per unit volume between the solid and liquid phases at the melting point.

We deployed both Stillinger-Weber ${ }^{18}$ (SW) and Tersoff-89 (Ref. 19) models to compute $\gamma_{\mathrm{sl}}$ of silicon. The reduced units of energy $(\varepsilon)$ and length $(\sigma)$ for SW model are $\varepsilon=3.4739 \times 10^{-19} \mathrm{~J}$ and $\sigma=0.2951 \mathrm{~nm}$, while for the Tersoff-89 model they are $\varepsilon=1.6022 \times 10^{-19} \mathrm{~J}$ and $\sigma$ $=0.1 \mathrm{~nm}$. Each MD time step corresponds to $0.5 \mathrm{fs}$. The simulations started with crystalline silicon structures of 5 $\times 5 \times 5$ cell units, which contain 1000 atoms, and a temperature of $1000 \mathrm{~K}$ for SW model and $2000 \mathrm{~K}$ for Tersoff- 89. Two heating rates were applied to the structure. The heating rate of $1 \times 10^{11} \mathrm{~K} / \mathrm{s}$ increase the temperature of the system by $12.5 \mathrm{~K}$ every 250000 steps, while that of $5 \times 10^{11} \mathrm{~K} / \mathrm{s}$ increases the temperature by $12.5 \mathrm{~K}$ every 50000 steps. The first 5000 steps after the heating was for equilibration, and thermodynamic data were measured for the remaining steps until the next temperature increase. The cooling process was performed when the temperature of the system reached $3000 \mathrm{~K}$ for SW and $4000 \mathrm{~K}$ for Tersoff- 89 model. The structure was then being cooled down to $500 \mathrm{~K}(1500 \mathrm{~K}$ for Tersoff-89) using the same rate as the heating. Potential energy and volume were recorded during the process in order 
TABLE I. Physical properties of silicon in the superheating process. Heating rate $(Q)$, superheating melting point $\left(T^{+}\right)$, melting point $\left(T_{m}\right)$, ratio of superheating meltion point to melting point $\left(\theta_{c}\right)$, nucleation barrier parameter $(\beta)$, average volume per atom $(V)$, enthalpy change per unit volume between solid and liquid states at melting point $\left(\Delta H_{m}\right)$, and solid-liquid interfacial tension $\left(\gamma_{\mathrm{sl}}\right)$ are displayed. The first two rows are results of the Stillinger-Weber model and the last two rows are those of the Tersoff- 89 model.

\begin{tabular}{lcccccccc}
\hline \hline & $\begin{array}{c}Q \\
\left(\times 10^{11} \mathrm{~K} / \mathrm{s}\right)\end{array}$ & $\begin{array}{c}T^{+} \\
(\mathrm{K})\end{array}$ & $\begin{array}{c}T_{m} \\
(\mathrm{~K})\end{array}$ & $\theta_{c}$ & $\beta$ & $\begin{array}{c}V \\
\left(\mathrm{~nm}^{3}\right)\end{array}$ & $\begin{array}{c}\Delta H_{m} \\
\left(\times 10^{9} \mathrm{~J} \mathrm{~m}^{-3}\right)\end{array}$ & $\begin{array}{c}\gamma_{\mathrm{sl}} \\
\left(\mathrm{J} / \mathrm{m}^{2}\right)\end{array}$ \\
\hline $\mathrm{SW}$ & 1.00 & 2338 & 1678 & 1.39 & 7.28 & 0.0196 & 2.64 & 0.412 \\
& 5.00 & 2388 & 1678 & 1.42 & 8.18 & 0.0196 & 2.64 & 0.429 \\
\multirow{4}{*}{ Tersoff } & 1.00 & 3260 & 2567 & 1.27 & 3.13 & 0.0206 & 3.31 & 0.417 \\
& 5.00 & 3220 & 2567 & 1.25 & 2.60 & 0.0206 & 3.31 & 0.392 \\
\hline \hline
\end{tabular}

to observe the superheating/undercooling temperature. The systems were run in isobaric-isothermal (NPT) ensemble by using the Nose-Andersen method. Pressure of the systems was set at zero for all simulations.

Silicon crystal undergoes superheating during the heating process and the structure melts at a temperature higher than the melting points (Table I). The melting points of the structure were determined by the coexisting solid-liquid phase method. ${ }^{20}$ The melting point of Tersoff- 89 model is $2567 \mathrm{~K}$, which is higher than both experimental value and the SW model. A potential energy of the system versus temperature curve shows that the system undergoes a phase transition at the superheating temperature $\left(T^{+}\right)$(Fig. 1). The ratio of superheating temperature to melting temperature is quite
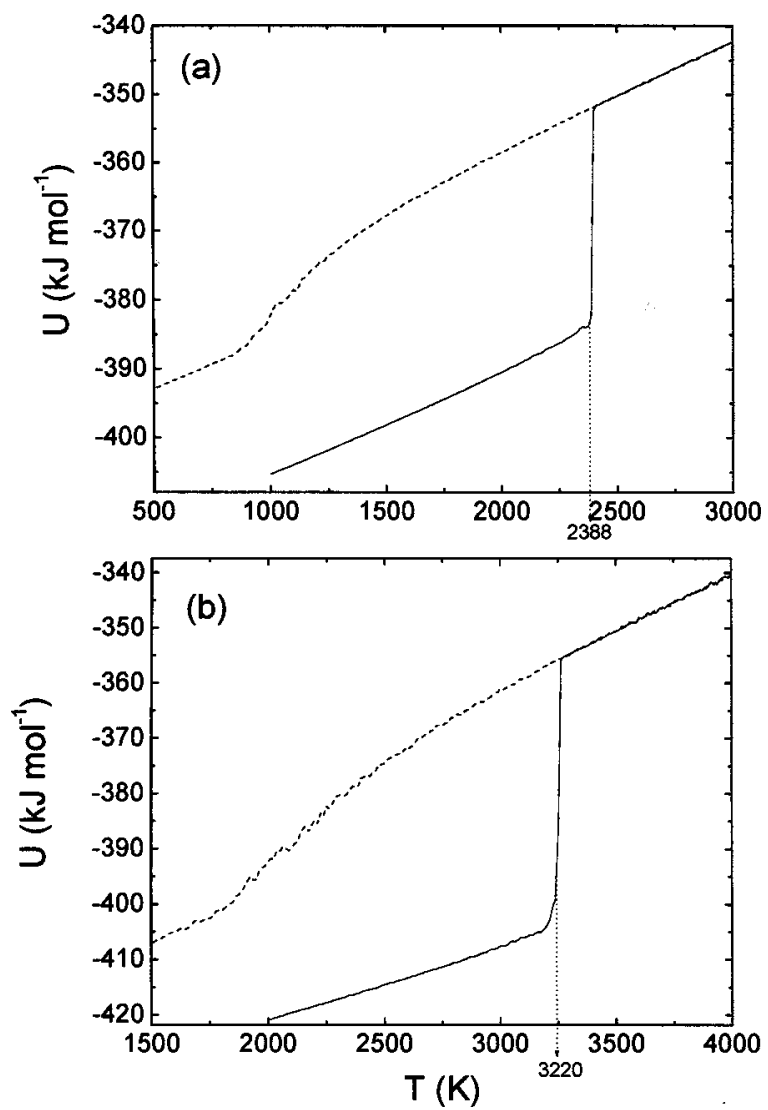

FIG. 1. Molar internal energy vs temperature during superheating (solid line) and undercooling (dotted line) process of silicon for (a) SW and (b) Tersoff- 89 models. The leaps in the superheating curves indicate a change of state. high due to the strong covalent bond in silicon crystal. During the cooling process, the potential energy and volume of silicon do not show a sudden change as observed in superheating, in part due to the use of rapid cooling rates. The volume of the system with increasing temperature is shown in Fig. 2. Similar to potential energy curves, a sudden drop of volume is observed when the crystalline structure of silicon breaks down. However, in the cooling process, the volume increases gradually with decreasing temperature during the transition. A larger fluctuation is observed with the Tersoff model [Fig. 2(b)], which is likely due to a much higher starting temperature than the SW model.

Once we calculated the nucleation barrier parameter $(\beta)$, we can determine the solid-liquid interfacial tension $\left(\gamma_{\mathrm{sl}}\right)$ by Eq. (2). Since the simulation was carried out at zero pressure, the enthalpy change at melting point is equal to the change of internal energy of the system. We calculate the difference of

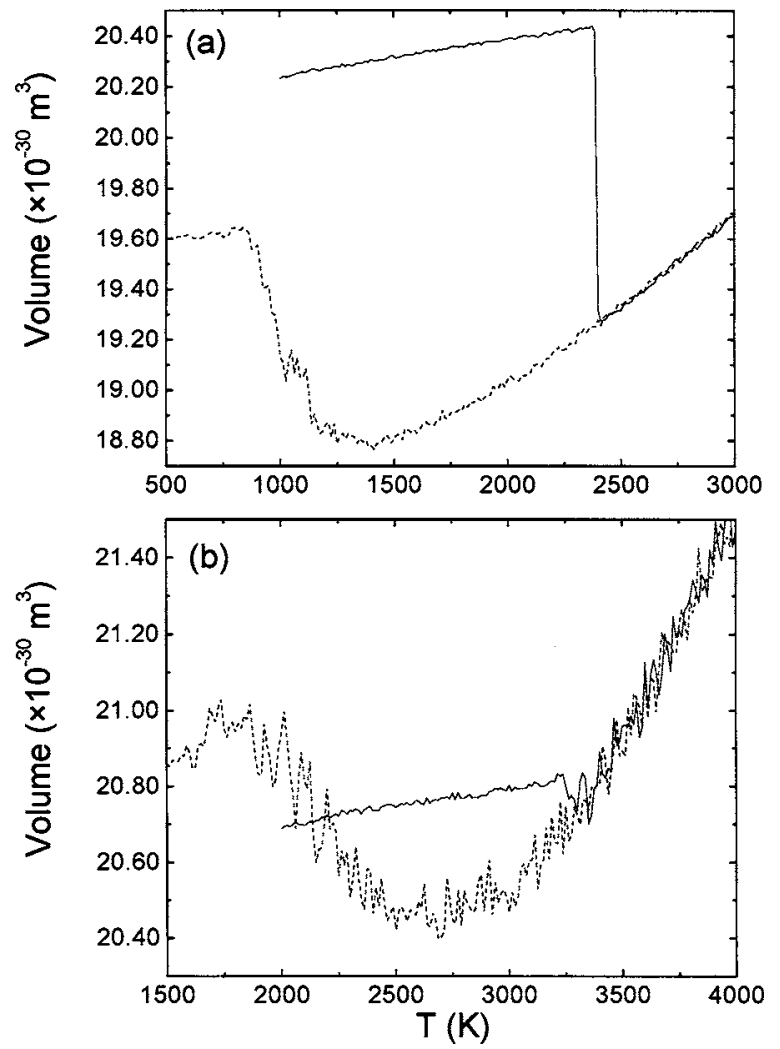

FIG. 2. Average volume per atom during the superheating (solid line) and undercooling (dotted line) process for (a) SW and (b) Tersoff- 89 models. 
enthalpy between the solid and liquid states at the melting point. We then divided the values with the mean molar volume of the solid and liquid to obtain the enthalpy change per unit volume at melting point. The average $\gamma_{\mathrm{sl}} \sim 0.413 \mathrm{~J} / \mathrm{m}^{2}$ (Table I), which is in good agreement with the measured result, ranging from 0.34 to $0.4 \mathrm{~J} / \mathrm{m}^{2}$. Moreover, the two silicon models give very close values of $\gamma_{\mathrm{sl}}$, even though the two models give dramatically different melting points. This suggests that the value of $\gamma_{\mathrm{sl}}$ is less sensitive to the model. Using a different heating rate also does not affect the final result of $\gamma_{\mathrm{sl}}$, as shown in Table I. Finally, we note that the $\gamma_{\mathrm{sl}}$ of SW silicon is about half the value of the liquid-vapor surface tension $\left(\sim 0.8 \mathrm{~J} / \mathrm{m}^{2}\right){ }^{9}$

In summary, we have deployed the superheating method of Luo et al. to compute the solid-liquid interfacial tension of silicon. Since there is no other computer simulation report on $\gamma_{\mathrm{sl}}$ of silicon, we hope that this piece of data can provide a useful benchmark on the interfacial properties of the two models of silicon. It is known that surface tension can be lowered appreciably when impurities (e.g., oxygen) exist in the structure. Research in this direction is under way.

The authors are grateful to valuable discussion with Dr. J. Morris. This work is supported by grants from the DOE (DE-FG02-04ER46164) and the Nebraska Research Initiative, and by John Simon Guggenheim Foundation and the
Research Computing Facility at University of NebraskaLincoln.

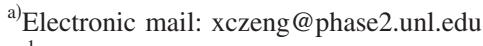

${ }^{1}$ S. R. Stiffler, M. O. Thompson, and P. S. Peercy, Phys. Rev. Lett. 60, 2519 (1988).

${ }^{2}$ A. J. Rulison and W. K. Rhim, Metall. Mater. Trans. B 26, 503 (1994).

${ }^{3}$ Y. Shao and F. Spaepen, J. Appl. Phys. 79, 2981 (1996).

${ }^{4}$ D. Li and D. M. Herlach, Europhys. Lett. 34, 423 (1996).

${ }^{5}$ T. Aoyama, Y. Takamura, and K. Kuribayashi, Metall. Mater. Trans. A 30, 1333 (1999).

${ }^{6}$ R. P. Liu, T. Volkmann, and D. M. Herlach, Acta Mater. 49, 439 (2001).

${ }^{7}$ G. Devaud and D. Turnbull, Appl. Phys. Lett. 46, 844 (1985).

${ }^{8}$ X. C. Zeng and D. Stroud, J. Phys.: Condens. Matter 1, 1779 (1989).

${ }^{9}$ Z. Q. Wang and D. Stroud, Phys. Rev. B 38, 1384 (1988).

${ }^{10}$ C. R. Miranda and A. Antionelli, J. Chem. Phys. 120, 11672 (2004).

${ }^{11}$ S. N. Luo, T. J. Ahrens, T. Çağin, A. Strachan, W. A. Goddard, and D. C. Swift, Phys. Rev. B 68, 134206 (2003).

${ }^{12}$ S. N. Luo, A. Strachan, and D. C. Swift, J. Chem. Phys. 120, 11640 (2004).

${ }^{13}$ S. N. Luo, A. Strachan, and D. C. Swift, Modell. Simul. Mater. Sci. Eng. 13, 321 (2005).

${ }^{14}$ W. D. Luedtke and U. Landman, Phys. Rev. B 40, 1164 (1989).

${ }^{15}$ S. Sastry and C. A. Angell, Nat. Mater. 2, 739 (2003).

${ }^{16}$ C. R. Miranda and A. Antonelli, J. Chem. Phys. 120, 11672 (2004).

${ }^{17}$ L. Zheng, S. N. Luo, and D. L. Thompson, J. Chem. Phys. 124, 154504 (2006).

${ }^{18}$ F. H. Stillinger and T. A. Weber, Phys. Rev. B 31, 5262 (1985).

${ }^{19}$ J. Tersoff, Phys. Rev. B 39, 5566 (1989).

${ }^{20}$ S. Yoo, X. C. Zeng, and J. R. Morris, J. Chem. Phys. 120, 1654 (2004). 